\title{
Akupunkturtherapie als Alternative zum Medikamenteneinsatz bei homöopathisch behandelten Patienten
}

\author{
Paul Rand \\ Praxis für Akupunktur-TCM (ASA) und klassische Homöopathie, Baden, Schweiz
}

D ie Reaktion des Patienten auf das korrekt verordnete homöopathische Mittel kann in den Begleiterscheinungen unangenehm sein. Gemeint ist dabei eine sehr intensive homöopathische Erstverschlimmerung wie auch andere Beschwerden in der Reaktionsphase der Behandlung (z.B. Auftauchen von alten Beschwerden, die in der Vergangenheit medikamentös statt geheilt nur unterdrückt worden sind). Bei sensiblen Patienten können solche Beschwerden (z.B. Schmerzen) ohne Hilfe unerträglich erscheinen. Konventionelle Schmerzmittel können die Wirkung der Homöopathie aber beeinträchtigen bis aufheben. Das kann zu Fehlschlüssen in der Beurteilung des Heilungsablaufes führen. Der Autor hat jahrelange und sehr positive Therapie-Erfahrungen sowohl mit der klassischen Homöopathie als auch mit der Akupunktur. Beide Therapieverfahren werden in der Regel nicht kombiniert, da sie, wie vermutet, unterschiedliche regulative Wirkungen haben und somit interagieren könnten. In Ausnahmefällen - wie oben erwähnt - konnte der Autor jedoch immer wieder die Akupunktur mit Erfolg als eine wirkungsvolle Alternative zu Pharmaka einsetzen. Dies soll im Folgenden anhand von zwei Fallbeispielen verdeutlicht werden. Voraussetzung für einen TherapieErfolg ist die fachgerechte Anwendung von beiden medizinischen Verfahren.

\section{Methode und Definitionen}

Die Fallaufnahme der Patienten erfolgte nach den Regeln der klassischen

Die Heilreaktion bei konstitutionell homöopathisch behandelten Patienten kann unterschiedlich starke Begleiterscheinungen zeigen. In Ausnahmefällen müssen zu deren Linderung auch konventionelle Pharmaka eingesetzt werden. Diese wirken jedoch symptomunterdrückend, was die Heilreaktion der Homöopathie beeinträchtigen kann. Nach der Erfahrung des Autors bietet sich in solchen Situationen der Einsatz von Akupunktur als Alternative zu Pharmaka an. Bei fachgerechter Anwendung wirkt Akupunktur ganzheitlich und dadurch nicht symptomunterdrückend. Ihre Wirksamkeit zeigt sich sowohl bei der Linderung der homöopathischen „Erstverschlimmerung" wie auch bei anderen Beschwerden, die im Verlaufe einer homöopathischen Behandlung auftreten können. Die vorliegenden Erfahrungen haben gezeigt, dass die Homöopathie in ihrer Heilwirkung durch die Akupunktur nicht beeinträchtigt wurde. Nach Ansicht und Erfahrung des Autors erscheint daher die Kombination von Homöopathie und Akupunktur in Ausnahmefällen als sinnvoll. Ausführlich beschrieben werden zwei Fälle von Patientinnen mit schwerer Neurodermitis.

Schlüsselwörter: Homöopathie, Medikamenteneinsatz, Akupunktur als Alternative

\section{Acupuncture Therapy as an Alternative to Pharmaceuticals for Homeopathically Treated Patients}

The healing reactions experienced by patients who are treated with constitutional homeopathic remedies may include a variety of strong effects. In exceptional cases patients must even take conventional prescription drugs to relieve such effects, but these suppress the symptoms and in turn can impair the healing reaction of the homeopathic remedy. Based on the author's experience, an alternative to pharmaceuticals is the use of acupuncture. When applied professionally, acupuncture works holistically and does not suppress the symptoms. Its effectiveness is shown by the fact that it can reduce the homeopathic "initial worsening" symptoms, as well as other afflictions that may arise in the course of homeopathic treatment. Experience showed that acupuncture in no way negatively affected the healing influence of homeopathic treatment. According to the author, homeopathy and acupuncture in combination thus make good sense in exceptional cases. Two cases of patients with serious neurodermatitis are described in detail.

Key words: Homeopathy, medication usage, acupuncture as alternative

Homöopathie [1,2,5]. Zur Repertorisation der aufgenommenen Symptome wurde das „Vithoulkas Experten System“ (VES) angewendet [1]. Die homöopathische „Erstverschlimmerung“, auch „Reaktionsphase“ genannt, wurde definiert als Verschlimmerung der Krankheitssymptome des Patienten nach der Einnahme des homöopathi- schen Mittels. Auf diese müsste eine entscheidende und deutliche Besserung des Krankheitsbildes folgen, im besten Fall eine Heilung. Die Tatsache, dass bei chronischen Krankheiten die Reaktionsphase aus mehreren Phasen bestehen kann, namentlich in Fällen, wo Symptome während längerer Zeit unterdrückt wurden, ist allgemein be- 
kannt [1,2,5]. Typisch, wie bei den hier beschriebenen Fällen, sind Rückenbeschwerden oder Kopfschmerzen, die vor Jahren mit Medikamenten behandelt wurden und jetzt wieder heftigst auftreten. Je nach der Mittel- und Potenz-Wahl und der Reaktionsfähigkeit des Patienten, die wieder durch seine allgemeine Verfassung und sein Krankheitsbild bestimmt werden, zeigte sich die homöopathische Reaktionsphase unmittelbar in den ersten 2 Tagen unspezifisch (Müdigkeit, starke Kopfschmerzen) resp. nach 10-14 Tagen oder auch später mit spezifischen Beschwerden (bekannt aus der Krankengeschichte). Die Rückkehr früherer Symptome galt als ein sicheres Zeichen, dass die Krankheit auf dem Wege der Heilung ist [1,2,5]. Angewendete C200-Potenzen waren von der Firma DHU-OMIDA Schweiz, die C10.000 von Schmidt u. Nagel, Genf, Schweiz.

Die Beschwerden der Erstverschlimmerung (bzw. Reaktionsphase) wurden mit der Akupunktur nur dann behandelt, wenn deren Intensität für den Patienten entweder unerträglich oder behindernd für den Berufsalltag erschien. Es handelte sich immer um Situationen, in denen der behandelnde Arzt „routinemässig“ Analgetika, nichtsteroidale Entzündungshemmer oder sogar Cortison einsetzen würde.

Die Zielsetzung der Akupunkturtherapie war die Linderung von aufgetretenen Beschwerden bis $\mathrm{zu}$ deren Akzeptanz seitens des Patienten. Der homöopathische Heilungsablauf sollte dabei so wenig wie möglich beeinflusst werden. Bei der Auswahl der Akupunkturforamina wurden die Gesetzmässigkeiten der Syndromdiagnose der Chinesischen Medizin angewendet [8,9]. Gleiches betrifft die Technik der Nadelung [10]. Die Behandlungen wurden wegen der „akuten Situation“ in der gleichen Woche durchgeführt (mit Nadeln vom Typ Seirin oder chinesische Nadeln Hwato (Spiralgriff) zum einmaligen Gebrauch).

\section{Fall 1: Neurodermitis}

\section{Anamnese und Untersuchung}

Patientin, geb. 1975, ledig, von Beruf Kindergärtnerin. Im November 2001
Eintritt in unsere Praxis wegen eines therapieresistenten Ekzems mit starkem Hautjuckreiz, unter dem sie seit ihrem 5. Lebensjahr leidet. Bei Geburt hatte sie Milchschorf. In den letzten Jahren auch Heuschnupfenbeschwerden (Rhinoconjunctivitis pollinosa). Die übrige Anamnese ergab mit 11 Jahren Pfeiffersches Drüsenfieber und eine seit Jahren zunehmende Infektanfälligkeit mit rezidivierenden eitrigen Tonsillitiden und Mittelohrentzündungen. Diese wurden immer wieder antibiotisch behandelt. Bekannt war auch eine Milchunverträglichkeit. Bereits im Säuglingsalter konnte sie die Muttermilch nicht vertragen. Leider blieb auch eine konsequente milchfreie Diät ohne Auswirkung auf den Zustand ihrer Haut. In der Familienanamnese allergische Prädisposition seitens des Vaters (Heuschnupfen), sonst keine Auffälligkeiten.

Die Therapie wurde seit der Kindheit mit äusserlichen Cortisonanwendungen durchgeführt. Verschiedene intrakutane und serologische Untersuchungen an der Dermatologischen Poliklinik des UniversitätsSpitals in Zürich blieben ohne Resultat für den klinischen Zustand der Patientin. Ihre Erkrankung wurde als nicht heilbar bezeichnet. Seit 1995 wurde vom UniversitätsSpital Zürich eine Dauerbehandlung bestehend aus intramuskulären Cortison-Depots $3 x$ jährlich empfohlen und durchgeführt, unterstützend wurden äusserliche Cortisonsanwendungen verordnet. Auch im Jahr ihres Eintritts in unsere Praxis (2001) hatte sie bereits 2x Cortisondepot erhalten. Therapeutisch nahm sie gegen den Juckreiz und bei aktuellen Heuschnupfenbeschwerden verschiedene Antihistaminika (zuletzt Zyrtec 2-3 mal/Woche). Die Patientin lebt seit einigen Jahren in einer festen und glücklichen Beziehung. Wegen der andauernden Cortisonbehandlung war sie sehr besorgt in Bezug auf ihre Zukunft und eine Familienplanung.

Untersuchung: Stark entzündete trockene Haut mit Schuppen und Kratzspuren, betroffen waren Gesicht, Hals, Décolleté, Ellenbogenfalten, infragluteal beide Oberschenkel sowie die beiden Kniekehlen (Abb. 1). Sonst ohne Auffälligkeiten.

\section{Homöopathische Fallaufnahme}

Die Patientin erweckte im Gespräch einen sehr liebevollen und sanften Eindruck. Sie war offen, kommunikativ und wiederholt unsicher bei der Auskunft (sie korrigierte sich selber oder stellte das Gesagte in Frage). Am auffallendsten waren ihre Eile und Nervosität und allem voran die Unruhe der Arme und Beine während des Gesprächs. Sie bewegte diese unaufhörlich und kratzte sich immer wieder an verschiedenen Stellen der Haut.

Sie bezeichnete sich als eine „sehr zappelige und unruhige Person“. Sie wäre sehr gerne von vielen Menschen umgeben und sie sei absolut kein Einzelgänger. „Ich kann keinem Lebewesen etwas antun, auch nicht einer Fliege oder Pflanze“. Sie möchte allen alles recht machen, andernfalls hätte sie sofort ein schlechtes Gewissen. Jemandem etwas abzusagen oder Nein sagen „wäre ein Horror für sie“. Sie leide sehr unter ihrer Haut und „kratze sich bis ins Blut“. Sie weiche dem Blick der anderen Menschen auf der Strasse häufig aus, weil sie sich für ihr Aussehen so schämt. Sie sei sehr schreckhaft, hätte Angst vor dem Neuen und mag nicht alleine sein. Entscheidungen zu treffen wäre nicht ihre „Stärke“. Ihr grösstes Problem sei ihr Zeitmangel - sie ist immer „gehetzt“, sie nimmt die Wünsche und Lasten von anderen auf sich und hätte kaum Zeit für sich selber. Die Ablösung von der Familie (sie wohnte zusammen mit dem Freund) fiel ihr anfangs sehr schwer. Als Hobby gibt sie Musik und das Trommeln auf den Ölfässern in einer Karibik-Band an.

Ihr Freund beschrieb sie als offen, kontaktfreudig, sehr verantwortungsbewusst, sehr anhänglich und unsicher und immer in „Eile“. Sie nehme extrem viel Rücksicht auf die Wünsche der anderen, die sie immer vor die eigenen stellt. Wenn sie den Wohnort wechseln müsste, wäre es ein grosses Problem, weil sie grosse Angst vor Neuem und Veränderungen hätte. Er beschrieb sie auch als sehr leichtgläubig.

Die Mutter betonte ihre liebe und aufopfernde Natur, ihren ganz grossen Familiensinn und ihre ausgeprägte Hilfsbereitschaft. Einmal hätte sie ihre Korsika-Ferien vorzeitig abgebrochen, 

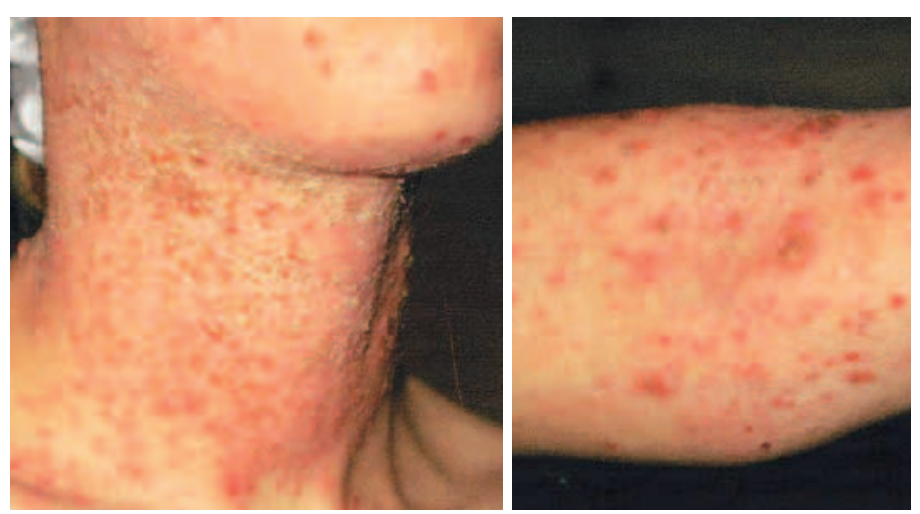

Abb. 1. Fall 1: Patientin mit Neurodermitis, Hautzustand vor Beginn der homöopathischen Therapie.

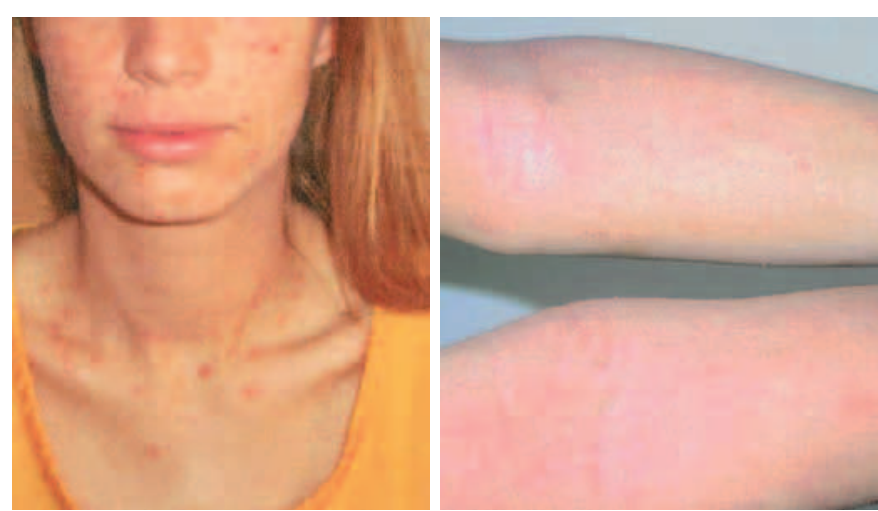

Abb. 2. Fall 1: Patientin mit Neurodermitis, Hautzustand 4 Wochen nach Beginn der homöopathischen Therapie.

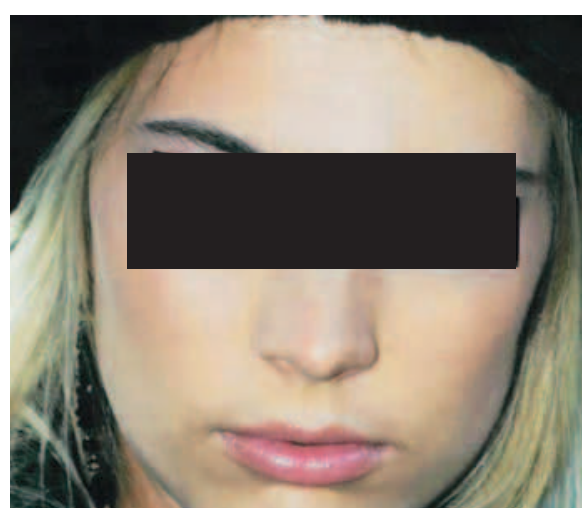

Abb. 3. Fall 1: Patientin mit Neurodermitis, Hautzustand nach Abschluss der homöopathischen Therapie.

als sie erfuhr, dass sich der 90-jährige Grossvater schlecht fühlt. Sie erzählte, dass ihre Tochter als Säugling die Muttermilch „im Schwall“ erbrach, weswegen sie sogar hospitalisiert werden musste. Am Ende betonte sie auch die ewige „Eile“, in der sich die Tochter immer befindet und vermutet die Ursache im „nie Nein sagen können“. Die vielen Verpflichtungen kosteten sie ihre ganze Freizeit. Sie betonte, wie nervös und doch sehr lieb ihre Tochter sei.

Weiterer Bericht der Patientin: Ihre Körperreaktion auf die Umgebungstemperatur hänge von dem aktuellen $\mathrm{Zu}$ stand ihrer Haut ab. Je mehr entzündet, desto weniger mag sie Sonne und Wärme. Ihre Hände und Füsse sind warm. Infolge der grossen Trockenheit der Haut schwitzt sie wenig, ausser gelegentlich in der Nacht. Der Schlaf ist unruhig und zusätzlich durch den Hautjuckreiz gestört. Deshalb ist sie häufig unausgeschlafen und müde. Die
Haut verschlechtert sich bei „Stress“ oder nervlicher Belastung. Nebenbei erwähnt sie eine schlechte Wundheilung, wo sie über ihre „Kratzspuren“ berichtet. Sie ist gewöhnt, wegen der Hauttrockenheit mehr zu trinken. Dadurch hat sie wenig Durst und wenn, dann eher abends zu Hause. Bei den Essgewohnheiten betont sie die Abneigung und Unverträglichkeit von Milch (Erbrechen und Durchfall), Erdnüssen, Zitrusfrüchten und Honig (Ekzemverschlimmerungen). Vorliebe empfindet sie für Süssigkeiten, Wild, Geräuchertes, bei Gelegenheit sogar für Speck (auf die Frage, ob sie Fettiges mag).

Ihre Periode ist seit Jahren durch die Pille geregelt. Sie führt ein erfülltes Sexualleben. Wenn die Haut sehr entzündet ist, leidet sie vermehrt unter vaginaler Trockenheit.

\section{Homöopathische Repertorisation}

Die ausgewählten Symptome, die repertorisiert wurden, waren mehrheitlich Gemütssymptome. Alleine diese hätten zur Wahl des Mittels wohl gereicht. Das Repertorisationsblatt ist komplett in Tabelle 1 zu sehen. Unsere Wahl fiel auf SILICEA.

\section{Therapie und Heilungsverlauf}

Als erster Schritt wurde der Patientin während der Periode die Pille abgesetzt. Daraufhin wurde sie aufgefordert, sich nach der ersten natürlichen Periode für die homöopathische Mitteleinnahme zu melden. Nachdem die Amenorrhoe bereits 2 Monate dauerte, wurde Silicea C200 (5 Globuli) verordnet, zumal dadurch die Amenorrhoe behoben werden müsste.
Etwa 24 Stunden nach der Einnahme des Mittels reagierte die Patientin mit derart starken Kopfschmerzen, dass sie unfähig war, ihrer Arbeit nachzugehen. Statt dessen meldete sie sich in unserer Praxis zur Akupunkturbehandlung. Dies führte nach einer Sitzung zum völligen Verschwinden der Kopfschmerzen, wodurch die Einnahme von Schmerzmitteln nicht nötig wurde.

Im weiteren Verlauf berichtete die Patientin über die Verschlimmerung des Ekzems, die 6 Wochen nach der Einnahme des Mittels erfolgte und für 10 bis 14 Tage bestehen blieb. $\mathrm{Zu}$ diesem Zeitpunkt bestellten wir die Patientin ein und mussten feststellen, dass sie trotz des schlechteren Zustandes ihrer Haut viel gefasster und auch ruhiger wirkte als früher. Auf die Frage nach ihrer Gemütsverfassung wurde diese Beobachtung von der Patientin bestätigt derart, dass sie sich „besser wehren kann als früher" und dass sie jetzt gegenüber dem Partner auch mal ihren Willen durchsetzen könnte.

Die deutliche Verschlimmerung der Haut bei Verbesserung des Gemüts wurde als typisches Zeichen der beginnenden Heilung bewertet $[1,2,4]$. Die Patientin wurde nach weiteren 6 Wochen einbestellt. Sie erschien aber bereits nach vier Wochen mit starken Lumbalgien. Der Hautzustand war zu diesem Zeitpunkt um einiges besser als beim Eintritt. Auch diesmal wurde Akupunktur eingesetzt und die Lumbago in zwei Sitzungen mit einem Abstand von 2 Tagen komplett behoben.

Der Heilungsablauf verlief in den folgenden Wochen unbehindert weiter 
Tab. 1. Fall 1: Patientin mit Neurodermitis, Repertorisationsblatt zur homöopathischen Therapie

Hinweise für die Verschreibung

Das wahrscheinlichste Arzneimittel ist sil. $\mathbf{8 1 1 . 2}$ (mit einer Sicherheit von 176 Punkten)

\section{Hinweise für die Befragung}

Diese Analyse umfaßt 17 einzelne Symptome und vergleicht die Muster von 305 Arzneimitteln

1. Gemüt - Milde

2. Gemüt - Nachgiebigkeit

3. Gemüt - Hast, Eile

4. Gemüt - Selbstvertrauen - Mangel an Selbstvertrauen

5. Gemüt - Liebevoll, voller Zuneigung, herzlich

6. Gemüt - Furcht - neuen Projekten, Unternehmungen; vor

7. Gemüt - Beschwerden durch - Erwartungsspannung

8. Gemüt - Gesellschaft - Verlangen nach - allein; agg. wenn

9. Gemüt - Beeindrucken, empfänglich für Eindrücke; leicht zu

10. Gemüt - Korrekt, anständig, wohlerzogen - zu

11. Extremitäten - Ruhelosigkeit

12. Allgemeines - Speisen und Getränke - Milch - agg. - Muttermilch

13. Allgemeines - Krankengeschichte von; persönliche - Tonsillitis; von wiederkehrender

14. Allgemeines - Speisen und Getränke - Milch - Abneigung

15. Allgemeines - Speisen und Getränke - Milch - agg.

16. Allgemeines - Speisen und Getränke - Honig - agg.

17. Allgemeines - Wunden - Heilungstendenz - langsam

$\begin{array}{rrr} & \text { sil. } \\ 2 & 3 & 92 \\ 3 & 2 & 31 \\ 3 & 3 & 124 \\ 3 & 3 & 96 \\ 2 & 2 & 54 \\ 2 & 2 & 5 \\ 2 & 3 & 74 \\ 2 & 1 & 48 \\ 2 & 2 & 45 \\ 1 & 1 & 3 \\ 3 & 3 & 71 \\ 3 & 3 & 4 \\ 2 & 2 & 18 \\ 2 & 2 & 72 \\ 3 & 1 & 99 \\ 2 & 2 & 5 \\ 2 & 3 & 56\end{array}$

(Abb. 2). Die psychische Verfassung der Patientin hatte sich so positiv entwickelt, dass Familie und Freund uns darüber telefonisch spontan berichtet haben. Nervosität, Schüchternheit, Unsicherheit, ständige Eile und Unruhe haben sich nach und nach gelegt. Bei den Konsultationen konnten wir feststellen, dass auch die Extremitätenunruhe verschwunden war.

Bis zur Heilung benötigten wir 10 Monate (Abb. 3). Die Amenorrhoe dauerte 6 Monate. Danach setzte eine regelmässige Monatsblutung ein. Die Patientin ist bis zum heutigen Tag ohne Symptome. Im Juni 2006 hat sie ihren Freund geheiratet und plant eine Familie.

\section{Einsatz der Akupunktur gegen die Beschwerden der Reaktionsphase: Kopfschmerzen}

24 Stunden nach der Einnahme von Silicea C200 bekam die Patientin starke Kopfschmerzen. Sie klagte über pulsierende Schmerzen in den Schläfen, oberhalb von den Augen und an der ganzen Schädeldecke. Es war ihr „trümlig“, sie hatte „Sturm im Kopf“, was wohl übersetzt mit Schwindelge- fühlen am ehesten $\mathrm{zu}$ beschreiben wäre. Begleitend Übelkeit ohne Erbrechen und phasenweise ein Gefühl von „Herzrasen“. Sie konnte die ganze Nacht nicht schlafen und fühlte sich sehr erschöpft. Obwohl sie Durst hatte, trank sie nur „schluckweise“, damit sie nicht erbricht.

Bei der Untersuchung war der Zungenkörper rot, trocken und ohne Belag. Der Puls war schnell und tief, der Leberpuls angespannt und die Nierenpulse beidseitig kaum vorhanden. Die festzustellende Syndromdiagnose: Leere-Hitze mit Trockenheit und Hitze des Blutes als Folge einer Nieren-YinLeere, die schliesslich zum aufsteigendem Leber-Yang führte [8,9]. Entsprechend die Foramina-Auswahl: Tai-Xi (Nieren 3), Shen-Shu (Blase 23), XingJian (Leber 2), Feng-Chi (Gallenblase 20) und He-Gu (Dickdarm 4), um das Nieren-Yin zu stärken, das Leber-Yang $\mathrm{zu}$ beruhigen und zu senken, Wind $\mathrm{zu}$ vertreiben und die Hitze zu kühlen $[7,8]$. Bereits während der Nadelung kam es zur deutlichen Linderung der Kopfschmerzen, die dann 2 Stunden nach der Behandlung gänzlich aufgehört haben.

\section{Lumbalgie}

Nach Ablauf von 3 Monaten kam die Patientin mit starken Kreuzschmerzen, die sich seit einigen Tagen entwickelt hatten. Sie war sich keiner Überanstrengung oder anderer Ursachen bewusst. Allerdings erinnerte sie sich bei dieser Gelegenheit, dass sie in den letzten 2-3 Jahren gehäuft ein „steifes Kreuz“ bzw. Rückenweh hatte. Die Ursache sah sie in der Überanstrengung im Beruf (Kindergärtnerin).

Die Untersuchung zeigte eine schmerzhafte muskuläre Verspannung im ganzen lumbosakralen Bereich mit massiver Einschränkung der LWS-Mobilität in allen Richtungen. Die Schmerzen beschränkten sich auf die lumbosakrale Region beidseitig ohne Ischialgie oder seitliche Betonung vom Schmerz. Sehr deutlich war die Druckempfindlichkeit im intervertebralen Raum L4/L5 und L5/S1. Es wurden keine neurologischen Ausfälle festgestellt (PSR, ASR, Sensibilität).

Die Zunge war gerötet, jedoch weniger als beim Eintritt. Belag fehlend. Der Puls war fein und leicht beschleunigt. Auffallend war die Besserung des Ekzems im Gesichts- und Hals-Bereich 
mit beginnender Besserung an den oberen Extremitäten. Die infragluteale Region und die Kniekehlen waren noch unverändert (homöopathisch gesehen ein Heilungsablauf nach dem Heringschen Gesetz „von oben nach unten“ und dadurch auch eine Bestätigung der korrekten Verordnung) [1,2]. Die Syndrom-Diagnose der Leere-Hitze bei der immer noch bestehenden Nieren-Yin-Leere blieb unverändert. Die Rückenbeschwerden der LWS, ein bekanntes und häufiges Begleitsymptom der Nieren-Leere, bestätigten das ganze Syndrombild $[8,9]$.

\section{Foramina-Auswahl und Behandlungsprinzip}

Behandelt wurden die druckschmerzhaften Punkte, die nach der Lehre der Chinesischen Medizin direkte Blockierungsstellen im Verlaufe der Akupunkturgefässe darstellen, Ming-Men (DuMai 4) und Yao-Yang-Guan (Du-Mai 3); dazu Shen-Shu (Blase 23) sowie Tai-Xi (Nieren 3) zur Stärkung der Niere bei der vorhandenen Nieren-Qi- und YinLeere; Da-Chang-Shu (Blase 25) als lokal auf die LWS wirkendes Foraminum und Wie-Zhong (Blase 40) als wirksames Foraminum für die ganze lumbosakrale Region [8,9].

Diese Behandlung führte mit zwei Akupunktursitzungen während 4 Tagen zur deutlichen Linderung der Schmerzen, die sich kurze Zeit darauf völlig zurückgebildet haben. Auch in diesem Fall konnte auf die Gabe von Schmerzmitteln verzichtet werden.

\section{Fall 2: Neurodermitis}

\section{Anamnese und Untersuchung}

Patientin, geb. 1967, verheiratet, Mutter von 2 Kindern, Hausfrau und Angestellte im Supermarkt. Eintritt in die Praxis im November 1996 mit der Diagnose „Atopisches Ekzem“, begleitet von starkem Juckreiz seit ihrem 10. Lebensjahr. Damals habe alles angefangen mit „offenen Achselhöhlen“. Mit der Cortisontherapie hat sich die Haut der Achselhöhlen beruhigt und die darauffolgende Zeit war ohne grössere Beschwerden. Mit 28 Jahren, kurz vor der Geburt ihrer Tochter, erneuter starker Ausbruch des Ekzems. Betroffen ist diesmal das ganze Gesicht, sehr stark beide Ohren, weiter der ganze Hals, Décolleté sowie die beiden Ellenbogen und Hände. Vom Hausarzt wurde sie zum Dermatologen und von diesem ins UniversitätsSpital Zürich (Dermatologische Klinik) geschickt. Alle Untersuchungen waren „ergebnislos“ und als Therapie wurde weiterhin Cortisonsalbe empfohlen. Wegen des starken Ekzembefalls der Gehörgänge mit Nässen und Krusten, die diese verstopften und Infektionen verursachten, musste sie immer wieder bei HNO-Ärzten mit Antibiotika (ATB) behandelt werden. Auch bei dieser Patientin gab es wiederholt eitrige Anginen in der Anamnese, die jeweils mit ATB behandelt wurden.

Die übrige Anamnese ergab sexuellen Missbrauch durch den Onkel vom 5. bis zum 10. Lebensjahr. Hinsichtlich dieser Zeit und des Ausbruchs des Ekzems (im 10. Lebensjahr!) ist der zeitliche Zusammenhang sehr auffallend. 1985 Appendicitis perforans, 1990 und 1995 Gestosis gravidarum in beiden Schwangerschaften. Allergie auf Wolle. In der Familienanamnese beim Bruder und Grossvater Psoriasis vulgaris und in der Verwandschaft Heuschnupfen.

Status praesens beim Eintritt: Auf den oben beschriebenen Körperregionen chronisch-entzündliche makulopapulöse Hauteffloreszenzen, bedeckt mit Schuppen. Beide äusseren Gehörgänge mit Schuppen verstopft, Ohrmuschel angeschwollen und teilweise nässend. Angeschwollene und ekzematös betroffene Augenlider. Kratzspuren an Hals, Ellbogenfalten und Händen (Abb. 4). Der übrige Status ohne Auffälligkeiten .

\section{Homöopathische Fallaufnahme}

Im Gespräch wirkte die Patientin etwas distanziert, sehr traurig und verschlossen. Auf die Frage, ob es besondere oder ungewöhnliche Ereignisse in ihrem Leben gab, erzählte sie zögernd und mit sichtlicher Mühe über den sexuellen Missbrauch durch den eigenen Onkel. Dieser hat nicht nur sie, sondern auch ihre Schwester missbraucht. Den Eltern hat sie dies erst mit 18 Jahren erzählt. Zu ihrer Enttäuschung haben diese aber „keine Konsequenzen“ daraus gezogen, was sie bis heute nicht verzeihen kann. Sie verspürt immer noch Wut vermischt mit Angst und Ekel, wenn sie an den Onkel denkt. Als dieser kürzlich einmal zufällig hinter ihr im Bus gesessen ist, musste sie aussteigen. Sie fügte $\mathrm{zu}$ : „Wenn ich an ihn denke, kommt mir das Wort „Schleim“ in den Sinn“. Auch heute noch ist sie sehr traurig darüber, was mit ihr geschehen ist.

Ihr Vater war zu den Kindern sehr streng und schlug manchmal auch die Mutter. Sie verspüre ihm gegenüber eine gewisse Abneigung. Sich selber bezeichnete sie als gefühlsvoll, einfühlsam und ziemlich sensibel, mit grossem Hang zum Perfektionismus. Bei Problemen „trägt sie diese lange in sich, bevor sie etwas sagt“. Ihre Ehe bezeichnete sie als „ruhig“. Ihr Ehemann ist anpassungsfähig und kompromissbereit. Sexuelles Verlangen z.Z. fehlend, ohne Abneigung gegen Koitus oder körperliche Berührung. Unehrlichkeit mag sie nicht und Ungerechtigkeit bereitet ihr grosse Mühe. Auf die Wetter-Frage sagte sie, dass es ihr "nicht wohl sei, wenn es heiss ist" und sie "flüchte sich immer in den Schatten“. Sonne direkt mag sie nicht. Ihr Schlaf ist gut, sie bevorzugt die linke Seite. Sie hat Angst vor Spinnen und grossen Käfern, vor Schlangen, vor Vergewaltigung, vor Dunkelheit und in der Menschenmenge.

Die Patientin hat wenig Durst, obwohl sie manchmal einen trockenen Mund hat. Sie ist relativ oft verstopft. Alkohol mag sie nicht. Sie isst keine fettigen Sachen, mag Fleisch nicht besonders, isst keine Muscheln, Langusten und Innereien, zeigt Abneigung gegen Milch und Eier, isst aber gerne süsse Kuchen. Sie liebt Salz, trinkt oft und viel salzige Bouillon und liebt gesalzene Pistazien. Früchte verzehrt sie regelmässig und gerne. Ihre Periode ist regelmässig, 28/7Tage, starke Blutungen. Vor der Periode gereizt, sonst o.B. Ihre Hobbies sind Handarbeiten und „schön Ordnung um sich haben“.

\section{Homöopathische Repertorisation}

Die ausgewählten Symptome und deren Repertorisation (Tabelle 2) ergaben NATRIUM MURIATICUM als Mittel der Wahl. 


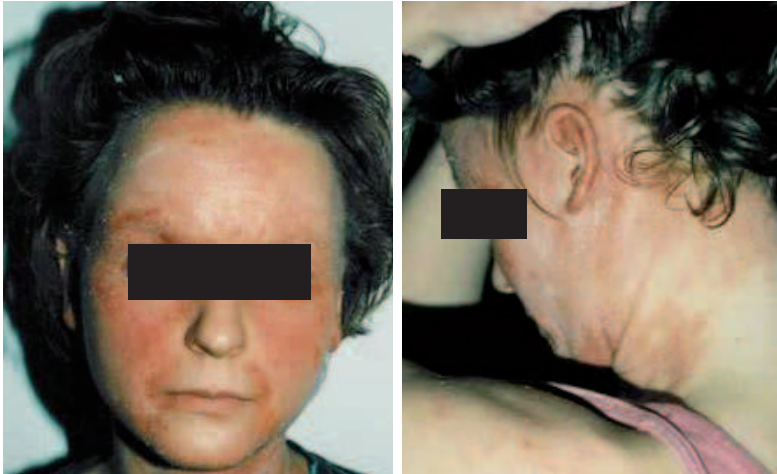

Abb. 4. Fall 2: Patientin mit Neurodermitis, Hautzustand vor Beginn der homöopathischen Therapie.

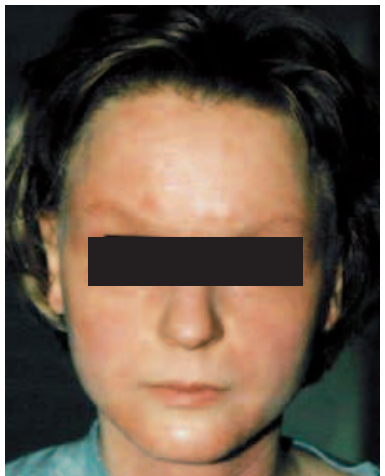

Abb. 5. Fall 2: Patientin mit Neurodermitis, Hautzustand 3 Wochen nach Beginn der homöopathischen Therapie.

\section{Therapie und Heilungsverlauf}

Die Behandlung wurde mit der Verordnung von Natrium muriaticum C200 begonnen. Die unmittelbare Reaktion waren Kopfschmerzen und Müdigkeit, die über 2 Tage andauerten und keine Gegenmassnahmen benötigten.

Nach Ablauf von 5 Wochen kam es zu einer Verschlimmerung des Ekzems mit unerträglichem Juckreiz und grosser Erschöpfung der Patientin. Die Haut war zerkratzt und die Patientin hat dringend Hilfe benötigt. Trotz der Situation war sie sehr tapfer und gefasst, voller Hoffnung und Vertrauen. Diese Tatsache, angesichts des wirklich sehr schlechten Zustandes der Haut, haben wir als „innere Besserung“ bei der „Verschlechterung nach Aussen“ bewertet. Mit anderen Worten, eine „klassische homöopathische Erstverschlimmerung“. Sie selber wollte zum Glück unter keinen Umständen Cortison, wollte kämpfen und akzeptierte gerne unseren Akupunktur-Vorschlag. Eine Behandlung hat den Juckreiz insofern gelindert, als dass die Patientin bereit war, eine weitere spontane Entwicklung abzuwarten. Nach einer Woche begann sich die Haut leicht zu bessern, was die Patientin zusätzlich sehr motivierte. Abb. 5 zeigt den nach 3 Wochen deutlich gebesserten Hautbefund. Bei genauerer Betrachtung vermittelte die Patientin auch allgemein gesehen einen besseren Eindruck als am Anfang. Die langsame Besserung setzte sich fort bis zu dem Zeitpunkt, als während unserer dreiwöchigen Ferienabwesen- heit vom HNO-Arzt bei der Infektion des Gehörganges Antibiotika verordnet wurden. Daraufhin kam es zur Hautverschlimmerung, jedoch längst nicht so stark wie beim Eintritt.

Unter der Vermutung einer antibiotikaverursachten Antidotierung des Mittels fiel die Entscheidung, eine Hochpotenz (C10000) zu verordnen. Grund dazu war auch die Tatsache, dass sich die Gemütsverfassung der Patientin verschlechterte und dass wir nach der C200-Potenz im seelischen Bereich doch mehr erwartet hatten, als tatsächlich geschehen ist. Das Thema des sexuellen Missbrauchs und ein „hintergründiger" stiller Kummer waren immer noch im Gespräch zu spüren und vorhanden.

Ende April 1997 erfolgte die Einnahme von Nat-m. C10000. Von diesem Tag an konnten wir bei den darauffolgenden Kontrollen auf allen Ebenen nur noch eine andauernde Besserung notieren. Die Ohren liessen wir durch den HNO-Arzt regelmässig reinigen. Nach dem Sommerurlaub berichtete die Patientin, dass sich auch ihr sexuelles Empfinden positiv verändert habe.

Im November 1997 wurde der Fall abgeschlossen. Zu diesem Zeitpunkt war die Haut bereits seit Wochen verheilt und das „Thema der Kindheit“ war verschmerzt. Bei der letzten Begegnung im Oktober 2004 war die Haut nach wie vor in sehr gutem $\mathrm{Zu}-$ stand (Abb. 6).

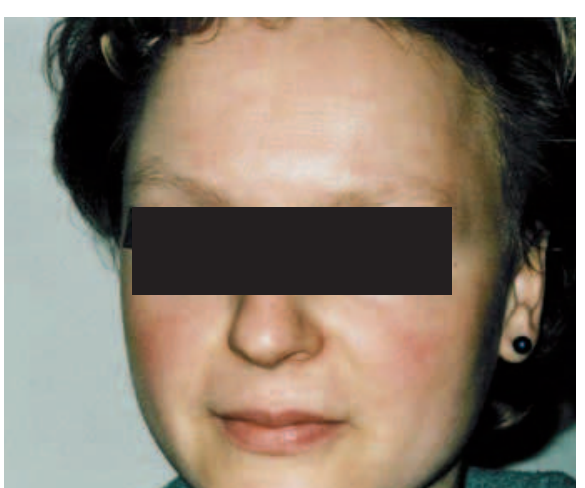

Abb. 6. Fall 2: Patientin mit Neurodermitis, Hautzustand nach Abschluss der homöopathischen Therapie.

\section{Einsatz der Akupunktur zur Linderung der homöopathischen Erstverschlimmerung: Ekzemverschlimmerung}

$\mathrm{Zu}$ der Hautreaktion kam es 5 Wochen nach der Einnahme von Nat-m. C200. Die Haut war an den Ekzemflächen stärker als am Anfang entzündet, schuppig, sehr trocken, mit noch mehr angeschwollenen Augenlidern und mit unerträglichem Juckreiz. Die Ohrenmuscheln waren stark entzündet und die Gehörgänge praktisch verstopft. Die Patientin kratzte sich unaufhörlich, was den Juckreiz nur noch schlimmer machte. Sie hatte einen trockenen Mund mit wenig Durst. Die Zunge war stark gerötet und ohne Belag (Befund der „reinen Zunge“ bei bestehender Obstipation führte uns zu der wertvollen Rubrik „Obstipation begleitet von reiner Zunge"; hier steht Nat-m. als einziges Mittel, und das im 2. Grad). Die gerötete und „reine“ Zunge führt zur Diagnose einer „Blut-Hitze“ $[7,10]$. Dem entsprach auch der Pulsbefund mit einem schnellen und feinen Puls. Bei der Hitze des Blutes sind die Patienten häufig ängstlich, so auch unsere Patientin (siehe homöopatische Fallaufnahme). Die Angst schwächt die Niere und die geschwächte Niere begünstigt die Enstehung von Furcht und Angst. Im Uterus verursacht die Blut-Hitze eine Hypermenorrhoe, die unsere Patientin seit Jahren hatte. Die Ursache für die entstandene Blut-Hitze ist ein Yin-Mangel, verursacht durch eine Nieren-Yin-Leere, wahrscheinlich entstanden durch die Ängste des jah- 
Tab. 2. Fall 2: Patientin mit Neurodermitis, Repertorisationsblatt zur homöopathischen Therapie

Hinweise für die Verschreibung

Das wahrscheinlichste Arneimittel ist nat-m. 570.9 (mit einer Sicherheit von 162 Punkten)

\section{Hinweise für die Befragung}

Diese Analyse umfaßt 22 einzelne Symptome und vergleicht die Muster von 504 Arzneimitteln

1. Gemüt - Verweilt - vergangenen unangenehmen Ereignissen; bei

2. Gemüt - Kummer - still

3. Gemüt - Verschlossen, zu

4. Gemüt - Heikel, pingelig

5. Gemüt - Ungerechtigkeit; erträgt keine

6. Gemüt - Abneigung - Familienangehörige; gegen

7. Gemüt - Furcht - Menschenmenge; in einer

8. Gemüt - Furcht - Dunkelheit, vor der

9. Mund - Trockenheit - Durstlosigkeit; mit

10. Rektum - Obstipation - begleitet von - Zunge - reine Zunge

11. Weibliche Genitalien - Sexuelles Verlangen - fehlend

12. Weibliche Genitalien - Menses - reichlich

13. Haut - Hautausschläge - juckend

14. Haut - Hautausschläge - trocken

15. Allgemeines - Speisen und Getränke - Eier - agg.

16. Allgemeines - Speisen und Getränke - Salz - Verlangen

17. Allgemeines - Speisen und Getränke - Fett - Abneigung

18. Allgemeines - Speisen und Getränke - Fleisch - Abneigung

19. Allgemeines - Speisen und Getränke - Alkohol - agg.

20. Allgemeines - Wetter - heißes Wetter - agg.

21. Allgemeines - Speisen und Getränke - Süßigkeiten - Verlangen

22. Allgemeines - Speisen und Getränke - Milch - agg.

\begin{tabular}{rrr} 
& \multicolumn{2}{r}{ nat-m. } \\
& I & \\
3 & 4 & 43 \\
3 & 3 & 34 \\
2 & 1 & 4 \\
2 & 2 & 48 \\
2 & 2 & 30 \\
1 & 1 & 24 \\
2 & 2 & 65 \\
1 & 1 & 61 \\
1 & 1 & 71 \\
2 & 2 & 1 \\
2 & 2 & 114 \\
2 & 3 & 281 \\
2 & 3 & 145 \\
1 & 1 & 72 \\
1 & 1 & 26 \\
3 & 4 & 84 \\
2 & 2 & 77 \\
2 & 2 & 139 \\
1 & 2 & 120 \\
2 & 2 & 36 \\
1 & 1 & 128 \\
2 & 2 & 99
\end{tabular}

relang sexuell missbrauchten Mädchens, das zusätzlich noch Angst vor dem strengen Vater hatte.

\section{Foramina-Auswahl}

\section{und Behandlungsprinzip}

Behandlungsprinzip: Blut beleben und kühlen, Wind-Hitze austreiben, NierenYin stärken.

Foramina-Auswahl: Qu-Chi (Di 11), $\mathrm{He}-\mathrm{Gu}$ (Di 4) vetreiben Wind-Hitze und kühlen das Blut, Xue-Hai (Mi 10) und San-yin-Jiao (Mi 6) kühlen und nähren Blut, Da Zhui (LG 14) vetreibt Wind und Hitze, Shen-Shu (Blase 23) ernährt Ni-Yin, Zu San Li (Ma 36) zum Ausgleich von Qi und Blut, harmonisiert Yin und Yang, tonisiert und stärkt Leere- und Schwäche-Zustände, stärkt Milz und Magen.

Diese Behandlung linderte den Juckreiz und die Entzündung und verhalf die schwierigste Phase der homöopathischen „Verschlimmerung“ ohne Pharmaka und Cortison zu überstehen.

\section{Diskussion}

Ein Versuch, die Reaktionen des $\mathrm{Pa}$ tienten auf das korrekt verordnete homöopathische Mittel zu unterdrücken, ist nicht nur untersagt, es könnte aus Sicht der klassischen Homöopathie sogar als Kunstfehler angesehen werden $[1,2,5,7]$. Bekannt ist, dass es grosse individuelle Unterschiede im Schmerzempfinden gibt. Es sind deswegen gerade diese sensiblen Patienten, die eine Problemgruppe und dadurch auch die Zielgruppe für die vorliegenden Untersuchungen darstellen. Der Einsatz von Pharmaka zur Linderung deren Beschwerden ist problematisch, denn Pharmaka können den Ablauf der homöopathischen Heilung unterdrücken oder beeinträchtigen - ein Dilemma, dem der Autor versucht, mit korrektem Einsatz der Akupunktur entgegen zu treten. Ermutigt dazu hat ihn die Tatsache, dass die Chinesische Medizin ein über Jahrhunderte erprobtes holistisches Heilsystem darstellt und nach Ansicht vieler Anwender keine Symptomunterdrückung verursachen soll. Auch in der Akupunktur gibt es das Phenomen der „Erstverschlimmerung“, auf die in der Regel eine Besserung des klinischen Zustandes folgt. Sind solche Paralellen in der Reaktionsphase der Akupunktur und Homöopathie nur ein Zufall? Das ist schwer zu glauben. Beide medizinischen Verfahren handeln anhand von streng definierten Gesetzen der Heilung und diese haben sich über Jahrhunderte bis Jahrtausende entwickelt $[1,2,5,7,8,9]$. Aus diesem Grund war der Autor davon überzeugt, dass die Akupunktur den angestrebten Heilungsablauf der Homöopathie in keiner Weise beeinträchtigen würde. Die bisherigen Erfahrungen sind positiv und unterstützten diese Annahme. So war es auch in den zwei ausgewählten Fällen von Neurodermitis, die in dieser Arbeit vorgestellt wurden. Auf den Medikamenteneinsatz konnte in beiden Fällen verzichtet werden. Die Akupunk- 
tur als Alternative zu Pharmaka hat nicht nur geholfen, sondern auch die homöopathische Heilung nicht beeinflusst.

Trotz der hier mit der Kombination von Akupunktur und Homöopathie beschriebenen ermutigenden Resultate ist noch Vorsicht geboten, solange nicht breitere Erfahrungen dokumentiert sind. Homöopathie und Akupunktur sollten im Allgemeinen nicht gleichzeitig angewendet werden. Beide Verfahren haben unterschiedliche regulative Wirkungen, könnten dadurch interagieren oder einander beeinflussen. Die Anwendung der Akupunktur bei homöopathisch behandelten Patienten sollte deswegen nur bei sensiblen Personen und dann nur als Alternative $\mathrm{zu}$ Pharmaka in Betracht gezogen werden. Gegenüber klassischen Pharmaka bietet Akupunktur folgende Vorteile:

- Die Akupunkturwirkung gliedert sich anhand der Syndromdiagnose kausal in das Krankheitsgeschehen ein.

- Der homöopathische Heilungsablauf wird durch die Akupunktur nicht beeinträchtigt.

- Akupunktur wirkt immunstimulierend und fördert dadurch die Heilung.
- Akupunktur wirkt nicht symptomunterdrückend.

- Akupunktur zeigt keine Nebenwirkungen oder Unverträglichkeitsreaktionen.

Die erfolgreiche Anwendung von Akupunktur und klassischer Homöopathie ist nur bei der entsprechenden Ausbildung des Arztes auf beiden Fachgebieten möglich. Im anderen Fall ist eine Zusammenarbeit zwischen den jeweiligen Fachärzten anzustreben. Zum jetzigen Zeitpunkt des Wissens und der Erfahrung sei nochmals betont, dass die Akupunktur bei homöopathisch behandelten Patienten lediglich als Alternative zum Medikamenteneinsatz zu betrachten ist. Die vorgelegte Arbeit möchte zur weiteren klinischen Forschung und Diskussion auf diesem Gebiet anregen.

\section{Fachliche Qualifikation des Autors:}

Facharzt für Innere Medizin, PhD (Prag), Facharzt FMH für TCM (ASA), Bachelor of Chinese Medicine (LICCM Basel), Absolvent und Diplominhaber der Internationalen Akademie für klassische Homöopathie (G. Vithoulkas) in Alonissos, Griechenland.

\section{Literatur}

1. Vithoulkas G: Vorlesungen an der Internationalen Akademie für klassische Homöopathie (1996-2000), Alonissos, Griechenland. Eigene Notizen des Autors.

2. Vithoulkas G: Die wissenschaftliche Homöopathie: Theorie und Praxis naturgesetzlichen Heilens, Ulrich Burgdorf Verlag für homöopathische Literatur, Göttingen 1986

3. Vithoulkas G: Materia medica viva, Urban und Fischer Verlag, 2000

4. Künzli von Fimmelsberg J, Barthel M: Kent’s Repertorium Generale, Barthel \& Barthel Verlag, Berg am Starnberger See, 1989

5. Kent JT: Zur Theorie der Homöopathie, 4. Auflage 1996, Karl F. Haug Verlag, Heidelberg.

6. Kent JT: Kents Arzneimittelbilder, 8. Auflage 1990, Karl F.Haug Verlag, Heidelberg.

7. Hahnemann S: Organon der Heilkunst, Karl F. Haug Verlag, Heidelberg 1996.

8. Schnorrenberger Claus C: Lehrbuch der chinesischen Medizin für westliche Aerzte, Hippokrates Verlag, Stuttgart 1983.

9. Schnorrenberger Claus C: Therapie mit Akupunktur Band I-III, Hippokrates Verlag, Stuttgart 1981, 1984, 1992

10. Schnorrenberger Claus C: Spezielle Techniken der Akupunktur und Moxibustion, Hippokrates Verlag, Stuttgart 1984

11. Schnorrenberger Claus C, Schnorrenberger B: Taschenatlas der Zungendiagnostik, Hippokrates Verlag, MVS Medizinverlage, Stuttgart 2002.

\section{Korrespondenzadresse:}

Dr. med. Paul Rand

Praxis für Akupunktur-TCM (ASA)

und klassische Homöopathie

Stadtturmstrasse 18, CH-5400 Baden

paulrand@dplanet.ch 